\title{
Folic acid and the prevention of neural tube defects
}

\author{
A population strategy is needed
}

Over three years have passed since an increase in the intake of folic acid among women planning a pregnancy was shown to prevent most neural tube defects. ${ }^{1}$ The evidence is conclusive; prevention is effective for women who have not had a pregnancy in which the fetus had a neural tube defect as well as for those who have. ${ }^{2}$ National authorities have recommended that women planning a pregnancy should increase their intake of folic acid..$^{3-5}$ The extra folic acid needed for a reasonable protective effect is $0.4 \mathrm{mg}$ a day, twice the current average dietary intake of $0.2 \mathrm{mg}$. If no screening was carried out for neural tube defects (with subsequent abortions) about 1500 affected children would be born each year in Britain. Over 1000 of these defects could be prevented by women consuming extra folic acid. Sadly, most people are still not aware of the importance of folic acid in the prevention of neural tube defects. ${ }^{67} \mathrm{~A}$ public health initiative is urgently needed; the tragedy of a pregnancy in which the fetus has a neural tube defect is even greater when it can be so easily prevented.

Some people argue that preventive strategies should be selectively targeted at women planning a pregnancy-on the grounds that only pregnant women and their children stand to benefit and only a few of these are affected. From a public health perspective, however, most disorders affect only a minority of people at risk and are often restricted to specific groups of the population (for example, children, pregnant women, or elderly people).

Immunisation programmes apply to all even though only a small fraction of the population is likely to benefit. The prevention of rubella in pregnancy to avoid the congenital rubella syndrome originally focused on two target groupsteenage girls, who were vaccinated at school, and pregnant women, who were screened and, if found to be susceptible, vaccinated after their pregnancy. This selective approach had only a partial effect. Aiming at vaccinating 10-14 year old girls kept a pool of infection among men and boys and younger girls. Vaccination against rubella is now offered to all, boys included. The population approach of collective action has been an essential part of most of the achievements in public health. This is the strategy needed to prevent neural tube defects.

Critics ask why some people should make changes to their lifestyle or diet that will benefit only others. Such an argument implies that we act selfishly and accept policies only if each of us benefits personally. Yet most people fall into at least one minority group. We take actions that can benefit others-and others take actions that can benefit us-provided that the risk to subgroups who might be harmed is minimal (or can be avoided by other means). If the only preventive measures introduced were those in which every individual to whom they were applied stood to gain we would have few preventive measures in place.

A selective public health strategy to prevent neural tube defects would require women planning a pregnancy to take extra folic acid; this is less likely to be successful than a population approach. The women would have to take capsules: trebling the dietary intake of folic acid would require changes unlikely to be achievable in practice with currently available foods. A woman would have to consume over eight glasses of orange juice, 10 servings of broccoli, three servings of brussel sprouts, or appropriate combinations of such foods to receive an extra $0.4 \mathrm{mg}$ of folic acid each day.

Furthermore, a selective policy would fail to prevent neural tube defects in unplanned pregnancies. The population strategy avoids the problems of the selective approach, and since the remedy-the consumption of extra folic acid-is simple and cheap the mass of people who do not benefit directly need be no cause for concern. ${ }^{8}$ Folate deficiency occurs in groups other than pregnant women, so the population approach offers the potential for wider benefits. The crucial point is that even in large doses folic acid has not been shown to cause harm. ${ }^{9}$ Concern has been voiced about two groups. Folic acid may prevent the anaemia of pernicious anaemia and so alter the presentation of the disease, but this problem can be resolved by educating doctors that macrocytic anaemia does not inevitably accompany the neuropathy. ${ }^{8}$ Folic acid may also counter the anticonvulsant activity of some drugs used in epilepsy; the effect of fortifying food would probably be negligible but higher doses of anticonvulsant drugs might be needed for some patients.

The solution, then, is to fortify food with folic acid. A staple food consumed in reasonably predictable quantities by almost everyone in the population needs to be chosen. It should then be possible to titrate the amount of folic acid added to achieve an extra average intake of $0.4 \mathrm{mg}$ folic acid in the population without many people getting too much or too little. Cereal grain flour (including wheat flour and corn flour) is a convenient choice. The United States Public Health Service supports this proposal, and a United Kingdom Expert Advisory Group recommended the wide fortification of bread with folic acid. 
Unfortunately, a voluntary system of fortifying food with folic acid leaves the initiative with the food manufacturers, who do not see themselves as the custodians of public health. Manufacturers respond to what the public wishes to buy. The general public, however, cannot be expected to have sufficient detailed knowledge of the nutrients in foods for particular groups of people to select specific foods to avoid particular diseases at particular times in their lives. The public may reasonably expect that such nutritional issues will be covered by a national dietary policy.

We believe that the rather loose voluntary recommendation on the fortification of bread should be strengthened into a compulsory policy; at the same time we think that the opportunity should be taken to select flour rather than bread for fortification. The fortification of flour at milling sites would be simpler and more economical than the fortification of bread at the much larger number of manufacturing sites.

Some people will wish to buy unfortified foods, and unfortified flour should continue to be available-as should bread made with unfortified flour. These unfortified products would be the exception and would require special labelling. Fortified flour or bread should be the norm and freedom of choice would be preserved.

White flour is already compulsorily fortified in Britain with two vitamins (niacin and thiamine) and two minerals (calcium and iron). The government is currently seeking opinions on revising the policy on fortification. It is suggesting that flour should be deregulated and that niacin and thiamine should no longer be added; this proposal is based on the absence in Britain of disease due to a lack of these two vitamins. The request for advice on this proposal provides public health professionals with an opportunity to recommend to the government the compulsory fortification of flour with folic acid. If this were done at a level that would increase average intakes by $0.4 \mathrm{mg}$ a day most of the 1500 or so neural tube defects each year could be prevented. If this opportunity is missed the costs-the needless suffering of seriously disabled children, unnecessary terminations of pregnancy carried out because of an antenatal diagnosis of a neural tube defect, and wasted financial resources-will be great.

\section{NICHOLAS J WALD}

Professor

CAROL BOWER

Visiting research fellow

Department of Environmental and Preventive Medicine,

Wolfson Institute of Preventive Medicine,

Medical College of St Bartholomew's Hospital,

London EC1M 6BQ

\footnotetext{
MRC Vitamin Study Research Group. Prevention of neural tube defects: results of the MRC vitamin study. Lancet 1991;338:132-7.

2 Wald NJ. Folic acid and neural tube defects: the current evidence and implications for prevention. In: Neural tube defects. Chichester: Wiley, 1994:192-211. (CIBA Foundation symposium 181.)

3 Expert Advisory Group. Folic acid and the prevention of neural tube defects. London: Department of Health, 1992 .

4 Centers for Disease Control. Recommendations for the use of folic acid to reduce the number of cases of spina bifida and other neural tube defects. MMWR 1992;41:1-7.

5 National Health and Medical Research Council. Revised statement on the relationship between dietary folic acid and neural tube defects such as spina bifida. 115th session. Australia: NHMRC, 1993.

6 Sutcliffe M, Schorah CJ, Perry A, Wild J. Prevention of neural tube defects. Lancet 1993;342:1174.

7 Clark NAC, Fisk NM. Minimal compliance with the Department of Health recommendation for routine folate prophylaxis to prevent fetal neural tube defects. Br $\mathcal{F}$ Obstet Gynaecol 1994;101: 709-10.

8 Dickinson J. Does folic acid harm people with vitamin deficiency? A critical examination of the evidence, relevant to fortification of cereal grain. $Q \mathcal{F}$ Med (in press).

9 Butterworth CE, Tamura T. Folic acid safety and toxicity: a brief review. Am $\mathcal{f}$ Clin Nutr 1989;50:353-8.
}

\section{Unified training grade}

\section{Many rivers still to cross}

The report of the working party on the unified training grade ${ }^{1}$ is the first piece of a jigsaw that will provide the picture for higher specialist training recommended in the Calman report. ${ }^{2}$ But even after elision of the registrar and senior registrar grades, in Britain, unlike in other members of the European Union, specialist training will comprise two grades: senior house officer and specialist registrar.

Entry to, assessment of progress through, and exit from the new specialist registrar grade will be managed by postgraduate deans in close collaboration with the medical royal colleges. Before the new grade is implemented satisfactory appointment systems and instruments for assessment will have to be developed and the curriculums for all specialties and other guidance will need to be published. All specialties are expected to begin transitional arrangements within one year of the commissioning date, but much more information, particularly the numbers for the grade in each specialty, is urgently needed. Some specialties are likely to make rapid progress to a steady state (all eligible career registrars having entered the grade) after commissioning; for others the transitional period may last several years.

Under the proposed transitional arrangements all current senior registrars are given the option of automatic entry to the grade, although the benefits of this option are unclear. When registrars are already too numerous for likely opportunities to become a consultant those meeting the criteria for entry may have an uncertain wait for entry and the award of a national training number, and no guarantee of more than two years' employment.
If the proposed transitional arrangements are rejected another option may yet need exploring: the establishment of a grade independent of current senior and career registrars. This process would take longer than the proposed arrangements and would be unfair to those specialties that have already balanced training and career grades.

The key to success of the new grade-and to the whole of the Calman report-is far greater increases in the numbers of consultants. The almost annual gift of pump priming for new posts, given by successive ministers for health, only marginally affects the target for expansion. A less dramatic but more effective means would be for purchasers of services during their negotiations with trusts to insist on an increase in the number of consultants in specialties in which numbers of career registrars exceed likely demand. Without this the many efforts to improve specialist training in Britain will have been for nought.

The fate of flexible training is the second major concern. The argument that doctors in flexible training can be treated similarly to those in academic and research posts is flawed. Doctors in flexible posts may train twice as long as those in full time posts and predictions of likely demand for consultants so far in the future is more difficult. What has been achieved in providing flexible training - not least, gaining its acceptance by the profession-could be lost.

Arrangements and strategies for assessing the progress of specialist registrars have yet to be agreed. The risk of a complex process, with little in common among specialties, must be avoided. Another risk is the creation of a bureaucratic 